\title{
Clinical Practice Guidelines: who needs them?
}

\author{
Sarah Marriott
}

\begin{abstract}
Clinical Practice Guidellines (CPGs) are systematically developed statements to assist practitioner and patient in clinical decistons about approprlate heatth care for specilic clinical circumstances. The Royal College of Poychiatrists CPG Programme aims to develop clinical guidelines which are scientifically valld and acceptable to those affected by them. Af the same time, CPGs must be responstve to advances in knowledge, and versatile enough for the demands of routine proctice. Their development involves a number of stages and a variety of methods, built into a cycle of evaluation and review. The Programme has established priortiles for clinical topics for CPG development through consultation with the mental health community. Welldeveloped CPGs would benefit clinicians, patients and purchasers of care. It is now important to appraise their ability to change clinical proctice, the aseociated direct and indirect coets, and their value as a medical fechnology. The clinical professions are in the strongest position to co-ordinate their development, and guide their evaluation.
\end{abstract}

The purpose of clinical work is to provide care which is appropriate to the needs of patients. Care is appropriate when the benefits to the patient outweigh the risks by such a degree as to make it worth giving. In the clinical setting, a guideline is a statement which describes appropriate care, and signals clinical actions which have an established link to a desired health outcome.

In developing such guidelines a number of issues must be considered. First, decisions about appropriateness must take account of different viewpoints. For instance, a practitioner requires evidence of the clinical effectiveness of a procedure, and a purchaser also needs evidence of its cost-effectiveness, and patients want to know their chances of 'feeling better' when it is completed. Better information is being called for, to inform the decisions of all those affected by health care.

Secondly, the appropriateness of care is not absolute, but changes with advancing knowledge. Few would now defend the use of barbiturates, or long-term benzodiazepines in the treatment of symptoms of anxiety and depression, or the use of insulin-induced coma for the treatment of a patient suffering from mental illness. New approaches are introduced because they are thought to have the potential to improve patient care. They are systematically evaluated in research and practice settings, and abandoned if it becomes apparent that their benefits do not outweigh the risks. Psychiatry, like all fields of medicine, is scattered with examples of treatment that have gone out of fashion.

Finally, clinical decision-making is a complex process, focusing on the individual needs of patients; for example, consider the clinical decisions involved in choosing a suitable management for a patient with schizophrenia who continues to hear frightening auditory hallucinations, despite an adequate trial of antipsychotic medication. The clinician may consider a social, pharmacological or psychological intervention, or combination of these in further management. The patient's preferences, together with those of the carers will be influential. If a pharmacological approach is appropriate, the patient's age, current health, past medical history, and immediate risk will influence the clinician's choice of the most suitable preparation from a range of anti-psychotic drugs and a safe, but effective dose range. For instance, the risks and benefits of using an atypical neuroleptic such as clozapine, will include evidence of its clinical effectiveness, as well as its resource implications, including assessment and monitoring procedures, compared against the same evidence associated with more traditional preparations. He or she will consider the availability of the necessary clinical skdlls and the competing needs of other patients. Social and psychological treatments will be appraised in the same way.

Given this complexity, it comes as little surprise that variation is found whenever patterns of care are compared across 
Table 1. The applications of CPGS

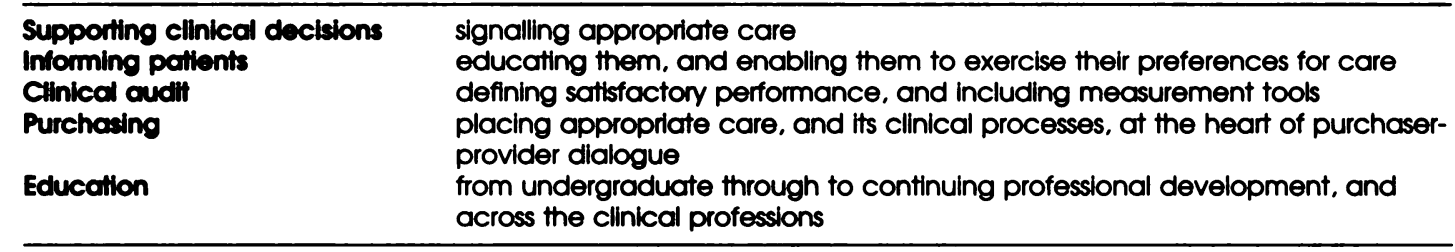

national, regional, and service boundaries. Examples in psychiatry include a twelve-fold variation in the use of ECT in two English regions (Pippard, 1992), and considerable diversity in approaches to the management of common conditions, such as disturbed behaviour in in-patients (Cunnane, 1994). Variation is a natural phenomenon, and not necessarily undesirable. However, where it remains unexplained it prompts concern that care is sometimes inappropriate.

To support clinical decision making statements of appropriate care must be scientifically valid, and acceptable to those affected by them. They must provide a framework which is responstve to advances in knowledge and understanding, and versatile enough to apply in the range of practice settings. Clinical Practice Guidelines are "systematically developed statements to assist patients and practitioners in clinical decisions about appropriate care in specific circumstances" (Field \& Lohr, 1990). To achieve each of these aims involves a number of stages, and a variety of methods built into a cycle of evaluation, implementation, review and revision.

The main purpose of well developed CPGs is to support clinical decisions. They can also contribute to clinical education, across professional groups, and to the development of audit tools for the collection of information about clinical practice for clinical audit. CPGs can inform the dialogue with purchasers about appropriate care for patients, and be used by clinicians to justify the resources needed to provide care. Finally, CPGs which define the clinical processes of appropriate care would inform the development of service specifications which support its delivery.

\section{The College CPG Programme}

During 1994, the Royal College of Psychiatrists commissioned a review of the development methods and potential applications of CPGs. This included an extensive literature review, and visits to groups in the UK and North America with expertise in developing and using CPGs. The findings were published as a College Council Report (Marriott \& Lelliott, 1994). In response to this the College CPG Office was established at the beginning of 1995. The programme's work is building on lessons learned by other professional organisations already developing guidelines, particularly the Agency for Health Care Policy and Research (AHCPR) in the USA, and the Practice Guidelines Resource Group in Canada, and the Scottish Intercollegiate Guideline Network (SIGN).

The Programme is led by a Steering Group. chaired by the Registrar of the College, which

Table 2. The framework of the College CPG Programme

\begin{tabular}{|c|c|}
\hline $\begin{array}{l}\text { Work group } \\
\text { Facilliator }\end{array}$ & $\begin{array}{l}\text { Promoting collaboration with other mental health groups } \\
\text { ldentifying prioritles } \\
\text { Co-ordinating the programme's work } \\
\text { Qually appralsal } \\
\text { Costing the programme } \\
\text { Agreeing project plans with indlividual work groups including methods for } \\
\text { appralsing evidence, and incorporating clinical and non clinical factors } \\
\text { Undertaking the development of indlidual CPGs in clinical areas prioritised } \\
\text { Working within the remit of the project plan } \\
\text { To support the Programme's work }\end{array}$ \\
\hline
\end{tabular}


co-ordinates the work of individual work groups. Each CPG will be developed by a separate work group following a project plan agreed with the steering group. While limiting the size of the work groups, their composition will reflect the interests of those affected by the guideline, and ensure the process involves all relevant clinical disciplines, users, carers and their advocates, and purchaser and provider managers where appropriate. A full-time facilitator will support the Programme's work.

The Programme will address each of the following stages in CPG development and implementation (Marriott \& Lelliott, 1994). Although described sequentially, the work of different stages will proceed simultaneously.

\section{Setting priorities across the mental health community}

An important first step for guideline developers is the fomulation of sensible priorities. The College Steering Group has recently consulted across the mental health community, commissioning a large national survey including psychiatric audit convenors, representatives from each of the professions involved in mental health, consultant and trainee psychiatrists from all the specialities, service users and their representatives, and provider groups. Preliminary findings suggest considerable agreement among them on priorities, and the full findings will be published soon. Clinical issues arising in the assessment and management of high risk behaviour will feature strongly in the first College CPG.

\section{Critical review of the scientific evidence}

The soundness and relevance of the scientific evidence relating to a topic must be evaluated and weighted. This evidence may include studies of different design and detail, both published and unpublished literature, and of varying degrees of rigour.

The College Programme will use a systematic approach to identify relevant literature, and grade it according to the study design it is derived from. Randomised controlled trials are the most powerful research design, but where they are not available there are strong arguments for the value of results from studies with less powerful designs.

The end products of this stage are provisional recommendations based solely on the research evidence.

\section{Clinical and non-clinical modulating}

factors

Clinical and non-clinical factors which modulate the scientific evidence are incorporated through a range of consultation strategies, tailored to the clinical area being considered. These may range from focused discussion in small 'expert' groups, to wider debate in conference settings, and structured postal consultation across the wider community. They may include the judgements of those who provide care, including consultant and trainee psychiatrists, other clinical professions, and the primary care team, those who receive care, including patients, carers and their advocates; and those who support care, including commissioners, and provider managers.

\section{Appraisal, review and revision}

Guidelines should be subject to external peer review to appraise the validity of their development and content. A number of tools have been developed to rate the important attributes of CPGs, including validity, reliability, and clarity. Only well-designed pilot trials can establish a CPG's clinical effectiveness.

\section{The CPG products}

Most guideline developers have chosen to design sets of guidelines, which provide a comprehensive overview of a clinical problem. For instance, the 1993 AHCPR guideline Depression in Primary Care: Detection, Diagnosis, and Treatment includes more than a hundred guideline statements, supported by 3,500 references. The parent product of CPG development can be large, and complex. This is called the Guideline Report.

Later products may have a variety of purposes, and may be presented as: a clinical tool - to support and inform clinical decisions; an audit tool - to yield valid, reliable, routinely collected data; an educational tool - to promote understanding; or a combination of these. The end products of a CPG can be presented in a wide variety of formats including structured medical records, pocket books summarising guidelines, computer help screens, and text books. Audit tools support the collection of measurement information by including clinical review criteria and clinical indicators. Educational tools may be targeted 
at a range of professional users, as well as presented as patient information.

\section{Getting guidelines into practice}

The main measure of success of a guideline is the extent to which it results in improved patient care in routine practice. To achieve this aim, clinicians must be aware of it, accept it and use it. Educational strategles are important in influencing clinicians' awareness, but are not necessarily sufficient to change clinical practice.

Barriers to implementing externally developed guidelines are often subtle, and can include an undermining of the sense of ownership among those who implement initiatives. Non clinical supports, such as information management systems, may also have a practical role to play in promoting, or hindering change. Patient specific reminders available at, or near to, the consultation appear to help clinicians to use guidelines. When guidelines are set out as broad statements of principle their operational detail can be developed by the clinicians who implement them, in accordance with local circumstance and need. Carefully implemented guidelines can change clinical practice, but more evidence is needed to understand their impact on health outcomes (Effective Health Care, 1994).

The College is perhaps well placed to help encourage the local use of CPGs. A number of existing structures can contribute to educating end users about CPGs, and how they can be used. The College is already supporting professional education, audit and research, as well as collaborating with other clinical organisations, and user and carer groups. Networks to local clinicians are established through the continuing professional development programme, the College Training Committee, the National Register of Audit Convenors, and the Examinations and Accreditation Committees. The College Research Unit has a track record of large-scale audit, and standard development projects.

\section{Conclusions}

A number of examples of centrally developed CPGs have already been published in Australia, North America and in Europe. Those developed by the Quality Assurance
Project in Australia, the American Psychiatric Association, and the AHCPR in North America, cover a range of mental health topics including the assessment and management of substance misuse disorders, eating disorders, depresstve disorders, schizophrenia and personality disorder. Closer to home, many national bodies have set up initiatives for guideline development including the Royal College of Nursing, the British Psychological Society, and each of the medical Royal Colleges. The opportunities for multidisciplinary collaboration are now considerable.

CPGs have wide implications, for practitioners of health care, as well as patients and purchasers. Their value as a clinical technology and their direct and indirect costs now warrant comprehensive evaluation (Feder, 1994). The clinical professions are in the strongest position to undertake their development, and guide this evaluation. The Royal College of Psychiatrists' first CPG will be available towards the end of next year.

\section{References}

Cunnane, J. G. (1994) Drug management of disturbed behaviour by psychiatrists. Psychiatric Bulletin. 18. 138-139.

Effective Health CARE (1994) Implementing Clinical Practice Guildelines: Can guidelines be used to improve clinical practice? Bulletin No. 8. Leeds: Untversity of Leeds.

FEDER, G. (1994) Clinical Guidelines in 1994: let's be careful out there. British Medical Journal, 309, 1457-1458.

FIELD, M. J. \& LOHR, K. N. (1990) Clinical Practice Guideline: from development to use. Washington DC: National Academy Press.

MARRIOT, S. \& LELWOT, P. (1994) Clinical Practice Guidelines and Their Development. Councll Report CR34. London: Royal College of Psychiatrists.

PIPPARD, J. (1992) Audit of electroconvulstie therapy in two NHS reglons. British Journal of Psychiatry, 160, 621637.

Sarah Marriott, Senior Registrar, CPG Office, Royal College of Psychiatrists, London SWIX 8PG and Maudsley Hospital, London SE5 8AZ

For further information about the Royal College of Psychiatrists CPG Programme, and the Audit and Information Service, please contact Ms Claire Palmer, CPG Facilitator, CPG Office, Royal College of Psychiatrists, London SW1X 8PG. 